\title{
Gemcitabine lipid prodrugs: the key role of the lipid moiety on the
}

\section{self-assembly into nanoparticles}

Eleonore Coppens, ${ }^{\S}$ Didier Desmaële, ${ }^{\S}$ Julie Mougin, ${ }^{\S}$ Sandrine Tusseau-Nenez, ${ }^{\varphi}$ Patrick Couvreur, ${ }^{\S}$ Simona Mura ${ }^{\S, *}$

${ }^{\S}$ Institut Galien Paris-Saclay, UMR 8612, CNRS, Université Paris-Saclay, Faculté de Pharmacie, 5 rue Jean-Baptiste Clément, F-92296 Châtenay-Malabry cedex, France

${ }^{\varphi}$ Laboratoire de Physique de la Matière Condensée (PMC), CNRS, Ecole Polytechnique, Institut Polytechnique de Paris, 91120 Palaiseau, France

* Correspondence should be addressed to:

Simona Mura, Ph.D.

Institut Galien Paris-Saclay, UMR 8612, CNRS, Université Paris-Saclay, Faculté de Pharmacie, 5 rue Jean-Baptiste Clément, F-92296 Châtenay-Malabry cedex, France

Email: simona.mura@universite-paris-saclay.fr

Keywords: Lipid prodrugs, gemcitabine, self-assembly, nanoparticles, drug delivery 


\section{Abstract:}

A small library of amphiphilic prodrugs has been synthetised by conjugation of gemcitabine (Gem) (a hydrophilic nucleoside analogue) to a series of lipid moieties and investigated for their capacity to spontaneously self-assemble into nanosized objects by simple nanoprecipitation. Four of these conjugates formed stable nanoparticles (NPs) while with the others immediate aggregation occurred, whatever the tested experimental conditions. Whether such capacity could have been predicted based on the prodrug physico-chemical features was a matter of question. Among various parameters, the hydrophilic-lipophilic balance HLB value seemed to hold a predictive character. Indeed, we identified a threshold value which well correlated with the tendency (or not) of the synthetised prodrugs to form stable nanoparticles. Such hypothesis was further confirmed by broadening the analysis to gemcitabine and other nucleoside prodrugs already described in the literature. We also observed that in the case of gemcitabine prodrugs, the lipid moiety affected not only the colloidal properties but also the in vitro anticancer efficacy of the resulting nanoparticles. Overall, this study provides a useful demonstration of the predictive potential of the HLB value for lipid prodrug NP formulation and highlights the need of their opportune in vitro screening, as optimal drug loading does not always translate in an efficient biological activity. 


\section{Introduction}

Clinical efficacy of even extremely promising pre-clinical drug candidates is often compromised by non-favorable physicochemical properties such as limited aqueous solubility, poor permeability, short half-life due to instability in biological media and/or rapid elimination. ${ }^{1}$ With the aim of overcoming these challenging issues, prodrug approaches have been largely investigated and their relevance is well confirmed by the observation that prodrugs represent up to $10 \%$ of all the new drug molecules approved by the FDA over the 2008-2017 period. $^{2}$

Prodrugs can be obtained by chemical modification of the parent molecule with small moieties (e.g., ionizable groups which increase aqueous drug solubility), ${ }^{3,4}$ but derivatization with lipids, ${ }^{4-6}$ peptides $^{7}$ and polymers ${ }^{4,8-10}$ has also been proposed. The latter enables to protect the drug from the degradation and/or to achieve a controlled and sustained drug release. ${ }^{11-13}$ Whatever the modification, it induces a loss of the drug activity, which is then recovered in vivo after enzymatic and/or chemical activation of the prodrug. ${ }^{12,13}$

Among the different types of prodrugs, lipid-based ones, in which the drug is linked to fatty acids, cholesterol derivatives, phospholipids are of particular interest. ${ }^{6}$ Not only are these lipids biocompatible, thus limiting insurgence of undesired toxicity, but they also could facilitate the crossing of biological barriers such as the blood-brain-barrier ${ }^{14}$ or the gastrointestinal tract, ${ }^{15}$ therefore leading to an efficient delivery to the target cells or tissues. To a certain extent the nature of the lipid moiety allows to modulate the fate of the prodrug upon administration by exploiting specific lipid metabolism pathways within the body. ${ }^{16,17}$ Thus, for instance, orally-administered drugs can be conjugated to triglycerides to either facilitate selective absorption into the lymphatic circulation for lymphocytes and lymph nodes targeting, ${ }^{18}$ or to rejoin the systemic circulation while by-passing the first-pass hepatic metabolism. ${ }^{15}$ 
Steroid prodrugs hold potential for cancer cells targeting by taking advantage of the accumulation of steroids, in particular cholesterol, in neoplastic tissues. ${ }^{19,20}$ Likewise, fatty acids-based prodrugs allow drug delivery to tumors, fatty acids being also key nutrients for cancerous tissues. ${ }^{6,21,22}$ Yet, opportune formulation may be needed to overcome the lack of aqueous solubility of these lipid prodrugs and to allow their systemic administration in a clinically acceptable solvent. In this context, combination with nanoscale carriers has been proposed and lipid prodrugs have been successfully loaded into liposomes, ${ }^{23-27}$ solid lipid nanoparticles, ${ }^{28-31}$ micelles ${ }^{32-34}$ or polymer nanoparticles (NPs). ${ }^{35-37}$

On the contrary to the above-mentioned strategies, in which the prodrug was encapsulated into other nanocarriers, we have taken advantage of the dynamically folded conformation of squalene, a biocompatible triterpene, for drug conjugation and spontaneous supramolecular assembly as nanoparticles. ${ }^{38}$ In the latter the drug-squalene conjugate was the only component of the formed nanoparticles, thus avoiding the use of additional materials (polymers, lipids etc.) and excipients (surfactants, sugars etc.). This resulted in a considerable increase of the drug loading and, thanks to a simplified composition, it could eventually facilitate scalability and introduction on the market. Thus, the linkage of squalene to various drugs resulted in nanoparticles of about $100 \mathrm{~nm}$ diameter and represented a versatile nanomedicine platform..$^{38,39}$

This approach has been first applied to gemcitabine (Gem) with the aim to overcome the major limitations of this anticancer nucleoside analogue (e.g., short half-life, poor diffusion into cells and induction of drug resistance), which all restrict pharmacological efficacy. ${ }^{38,40}$ Conjugation to squalene advantageously modified the Gem pharmacokinetics, leading to a better therapeutic index compared to the free drug, in several experimental tumor models in mice and rats. $^{41-44}$ 
Despite these interesting results, whether the squalene was the only lipid able to combine both spontaneous self-assembly of the gemcitabine prodrugs and biological activity was still a matter of question. We already investigated the influence of the isoprene chain length, revealing that at least four isoprene units were required to formulate prodrug nanoparticles without additional excipients. $^{45}$

In the current study we extended the nature of the investigated lipid molecules and we included in our pool (i) the cholesterol, (ii) the alpha tocopherol, a fat-soluble vitamin, (iii) a few fatty acids and (iv) a diglyceride. We assessed the X-ray diffraction patterns of the synthetised Gem prodrugs and we evaluated their capacity to self-assemble as nanoparticles in pure water at the minimum concentration of $1 \mathrm{mg} \cdot \mathrm{mL}^{-1}$, necessary for further biological studies. When they were obtained, the resulting nanoparticles underwent a detailed physicochemical characterization before being investigated for their in vitro cytotoxicity on the MDA-MB-231 breast cancer cell line. The hydrophilic-lipophilic balance (HLB) of the prodrug library was used to assess whether self-assembly failure of some prodrugs into nanoparticles could have been predicted ahead of the time-consuming chemical synthesis and physico-chemical evaluation.

\section{Results and discussion}

\section{Synthesis of the lipid gemcitabine bioconjugates}

Using as reference the squalene moiety $\left(\mathrm{C}_{30} \mathrm{H}_{50}\right)$ characterized by 6 double bonds symmetrically disposed around the $\mathrm{C}_{12}-\mathrm{C}_{13}$ linkage, a series of lipids displaying different chemical features was chosen to synthetize a library of gemcitabine prodrugs (Figure 1). Thus, within the terpene family, the gemcitabine was conjugated to the perhydrogenated SQ (RSQ) devoid of unsaturation, as well as, to the solanesol (Sol), a long-chain polyisoprenoid alcohol incorporating nine isoprene units. Then, polycyclic structures were introduced by conjugation to cholesterol (Chol) and alpha-tocopherol (VitE), which possess a sterol and a 
chromanol carbon skeleton, respectively. Prodrugs derived from non-terpenoid lipids without lateral methyl groups were synthetised by gemcitabine linkage to fatty acids, both saturated (i.e., octacosanoic acid, C28) and unsaturated (i.e., oleic (OA), linoleic (LA), eicosapentaeneoic (EPA) and docosahexaenoic acid (DHA)). Last, by derivatization with the glyceryl 1,3-dipalmitate (Digly), a gemcitabine prodrug with two side chains was obtained. As previously reported for the squalene-gemcitabine prodrug (SQGem, Table 1, \#01), ${ }^{38}$ the different derivatives were synthetised by covalent conjugation of the lipid moieties onto the 4'-amino group of the cytosine nucleus of the drug as depicted in Figures 2-4. Conjugation on this position allowed to prevent the rapid drug degradation into the inactive difluorodeoxyuridine metabolite by action of the endogenous deaminases. ${ }^{43}$ For sake of comparison, aiming to have conjugates in which the lipid is anchored to the drug via an amide bond, the synthetic strategies have been opportunely adapted according to the functional group available on the former. When necessary, a glutaryl spacer was introduced between the lipid moiety and the amino group. Hence, lipids bearing a carboxylic acid function were first activated into the mixed anhydride using ethyl chloroformate and then reacted with the gemcitabine base (Figure 2) or its bis 3',5'-tert-butyldimethyl silyl protected form (Figure 3). The release of gemcitabine from these prodrugs is expected to occur in vivo after proteolytic cleavage of the amide bond by the lysosomal cathepsins (B and D), as already demonstrated for a variety of amide-bearing prodrugs ${ }^{46,47}$ including the squalene gemcitabine conjugate. ${ }^{38}$ Overexpression of these enzymes in several malignancies compared to healthy tissues ${ }^{48}$ would favor selective and controlled drug release at the tumor site. 


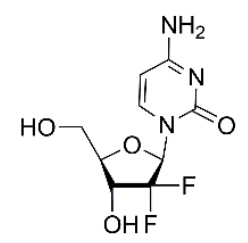

Gemcitabine

Terpenoids

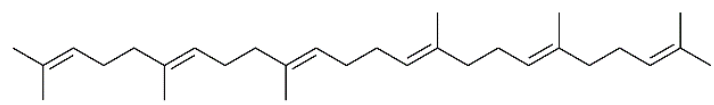

Squalene (SQ)

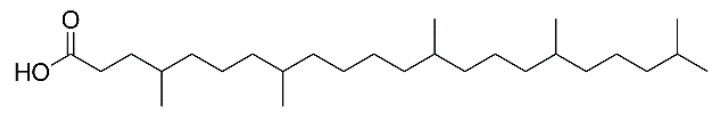

Saturated Squalenic acid (RSQ)

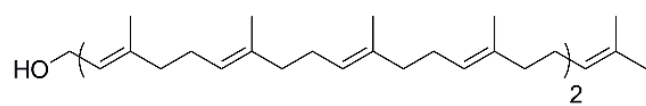

Solanesol (Sol)

Sterols

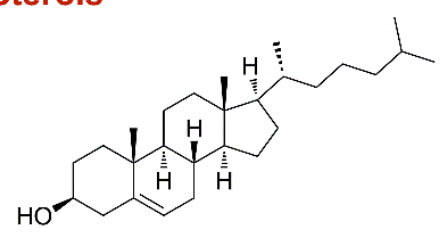

Cholesterol (Chol)

Fat-Soluble vitamins

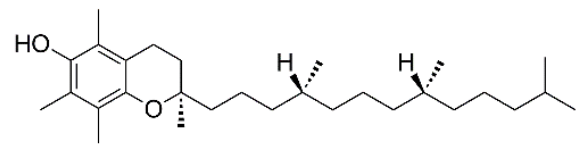

Alpha-tocopherol (VitE)
Diglycerides

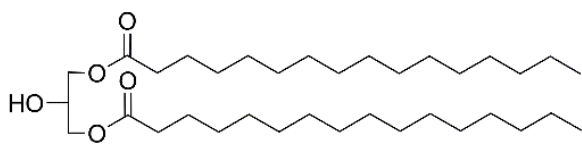

Glyceryl 1,3-dipalmitate (Digly)

Unsaturated fatty acids

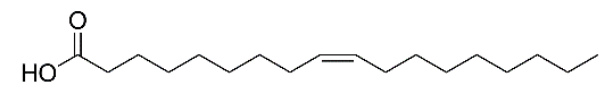

Oleic acid (OA)

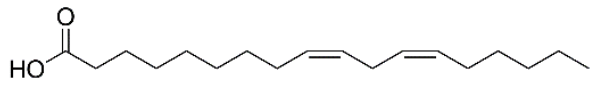

Linoleic acid (LA)

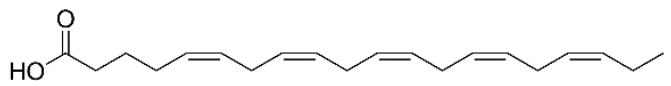

Eicosapentaeneoic acid (EPA)

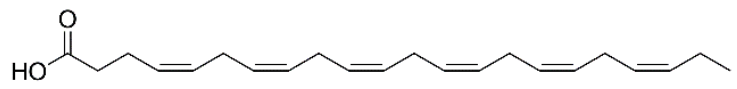

Docosahexaenoic acid (DHA)

Saturated fatty acids

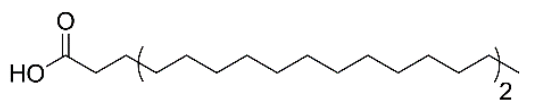

Octacosanoic acid (C28)

Figure 1. Structure of the gemcitabine and of the lipids used for the synthesis of the prodrugs. 


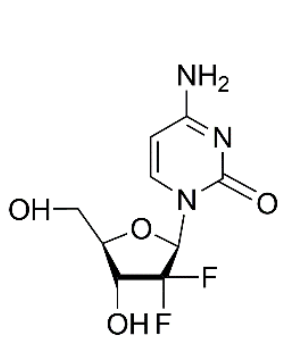

(Gem)

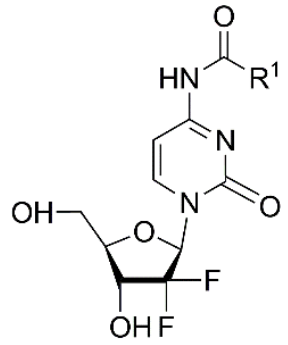

$$
\text { ( } \mathbf{R}^{1} \text { Gem) }
$$

$\# 01, \mathbf{R}^{1}=$<smiles>CC(C)=CCC/C(C)=C/CC/C(C)=C/CC/C=C(\C)CC/C=C(\C)CCC(C)(C)C</smiles>

$\# 02, \mathbf{R}^{1}=$

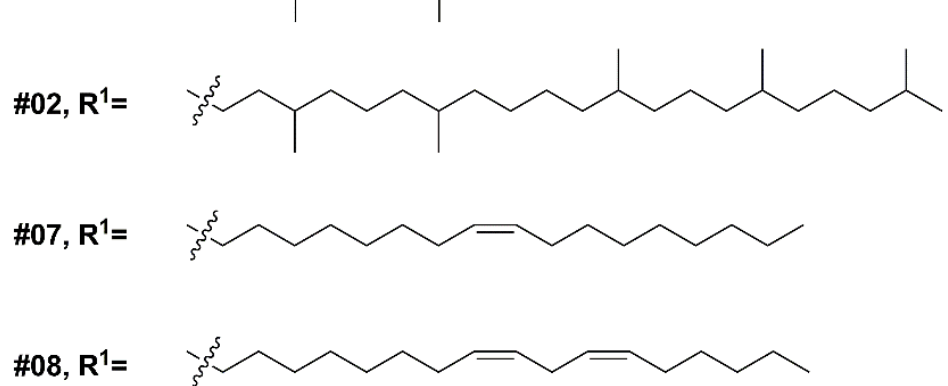

$\# 07, \mathbf{R}^{1}=$

$\# 08, \mathbf{R}^{1}=$

ethyl chloroformate, triethylamine THF, DMF

RT, 3-6 days

$\# 09, \mathbf{R}^{1}=$

$\# 10, \mathbf{R}^{1}=$

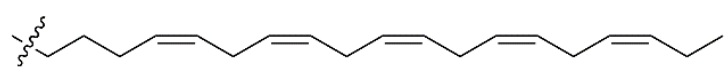

Figure 2. Synthetic strategy implemented to obtain the lipid prodrugs when using lipid moieties bearing a carboxylic group without protection of the gemcitabine moiety.

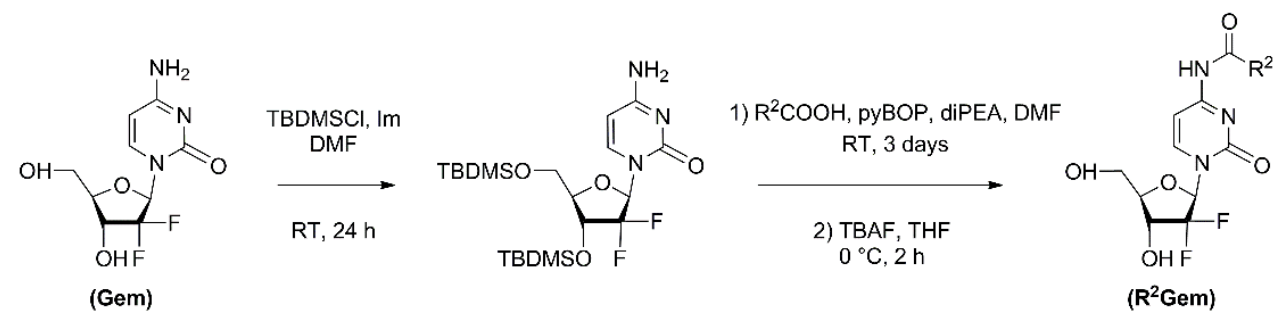

$\# 11, \mathrm{R}^{2}=$

Figure 3. Synthetic strategy applied to obtain the C28Gem lipid prodrug. 
Lipids bearing an alcohol function were first esterified with glutaric anhydride to provide the corresponding hemiglutaryl acid, which was then activated with ethyl chloroformate as described above or directly coupled using pyBOP as activating agent (Figure 4).
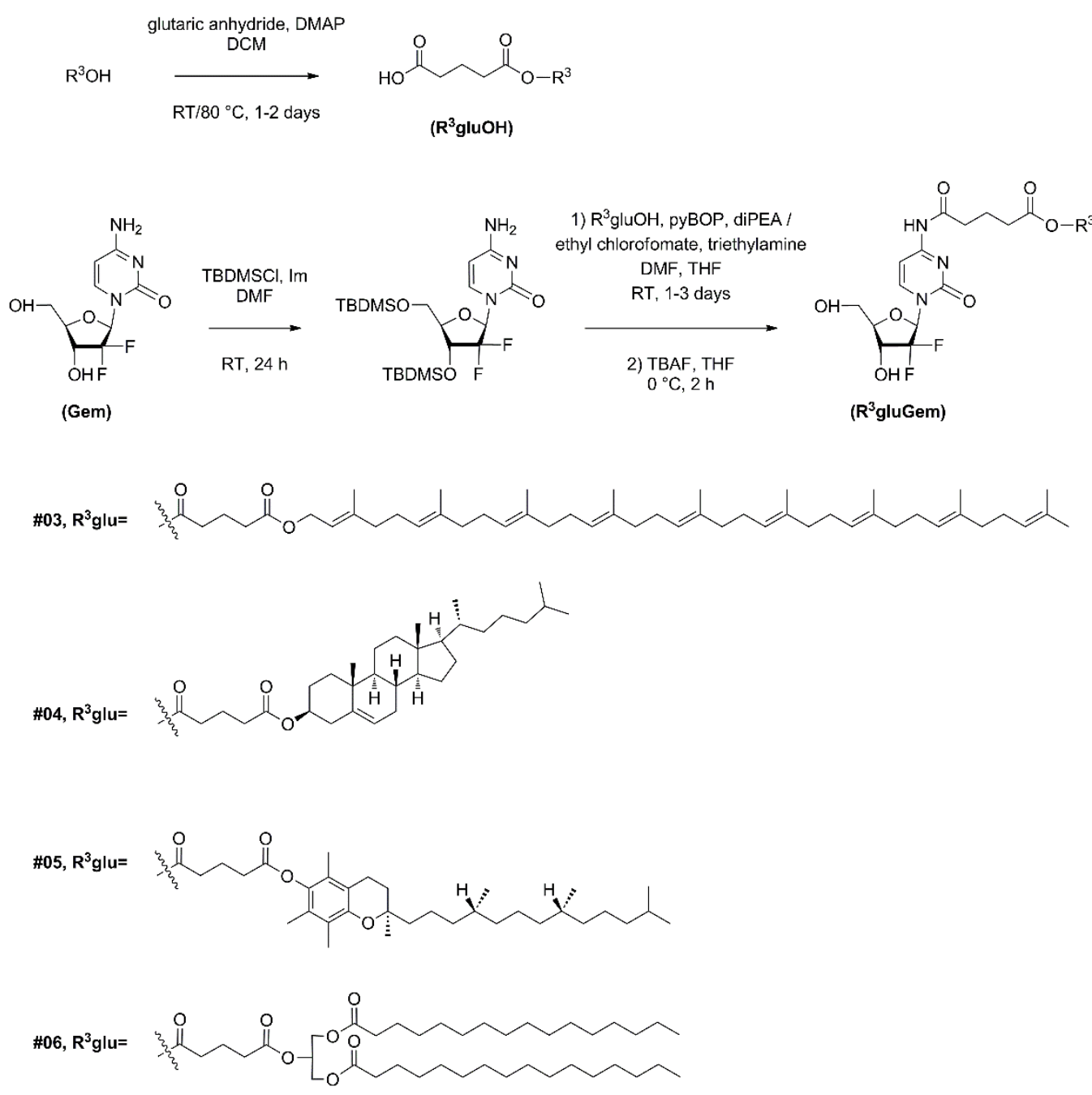

Figure 4. Synthetic strategy applied to obtain the lipid prodrugs while using lipid moieties bearing a $\mathrm{OH}$ group.

\section{Powder x-ray diffraction analysis}

Crystallinity of the synthetised prodrugs has been investigated by powder X-ray diffraction and $d$-spacing values are reported in Table S1. A similar pattern was observed for the SQGem 
and RSQGem prodrugs. At low angles, both conjugates showed two sharp peaks (a main one and one on the right), corresponding to first and second orders of the successive (001) Bragg reflections of a lamellar structure of $d$-spacing $44.5 \AA$ and $47.3 \AA$ for SQGem and RSQGem respectively (Figure 5a, Table S1). The shoulder observed on the left ( $d$-spacing $46.6 \AA$ and $50 \AA$ ) revealed the coexistence of two lamellar phases in each prodrug, as previously reported. ${ }^{49}$ In addition, a minor peak, corresponding to a $d$-spacing of about $40 \AA$, was found on the right of each first order peaks (Figure 5a). Overall, these results indicated that these two prodrugs could organise in a ternary mixture. At higher angles, both prodrugs revealed a broad peak centred at $4.8 \AA ̊$ (Figure 5b, Table S1).

Low angle XRD patterns of the other prodrugs revealed broad peaks, with variable intensity (Figure 5c, Table S1). Whereas SolgluGem (magenta line) and C28Gem (green line) exhibited (001) Bragg lines up to order three and two respectively, for the other prodrugs only a single broad peak (order 1), with $d$-spacing values comprised between $44.1 \AA$ and $51.5 \AA$, was observed. All these prodrugs showed a one-dimensional lamellar crystalline packing, and the width of the peaks can be correlated with the inability of these compounds to achieve a well-ordered long-range periodicity. SolgluGem (magenta line) and DiglygluGem (aquamarine line) presented the highest lamellar $d$-spacing values (67 $\AA$ or more), characteristic of an interdigitated lamellar gel phase $\mathrm{L}_{\beta} \cdot{ }^{50}$ At higher angles (Figure 5d, Table S1), apart from the C28Gem (green line), these prodrugs showed a very broad peak with $d$ spacing comprised between $5 \AA$ and $4.5 \AA$ and a shoulder at around $4.3 \AA<d$-spacing $<4.0$ $\AA$. The existence of several other peaks indicated the presence of one or more crystalline phases within the planes of the lamella, as also previously reported. ${ }^{49,51,52}$

It must be noted that at high angles, the whole library showed a broadening of the peaks which reflected the mean spacing of chains devoid of long-range order in the plane of the bilayer. 
Overall, a one-dimensional lamellar crystalline packing (Lc) was observed for all the prodrugs. When compared to other structural studies of gemcitabine lipid prodrugs, ${ }^{53}$ lower $d$ spacing values were herein observed, likely due to the presence of not fully extended and more disordered hydrocarbon chains. ${ }^{49}$ At higher angles, this disorder correlated with a main broad peak with a typical $d$-spacing $=4.6 \AA$, as already described for lecithins ${ }^{54}$ and stacks of sphingomyelin-cholesterol bilayers. ${ }^{55}$ The right asymmetry of these peaks can be modelled by a broad peak centred around $4.2 \AA$, corresponding to hexagonally packed hydrocarbon chains in gel phase. ${ }^{50,54,56}$
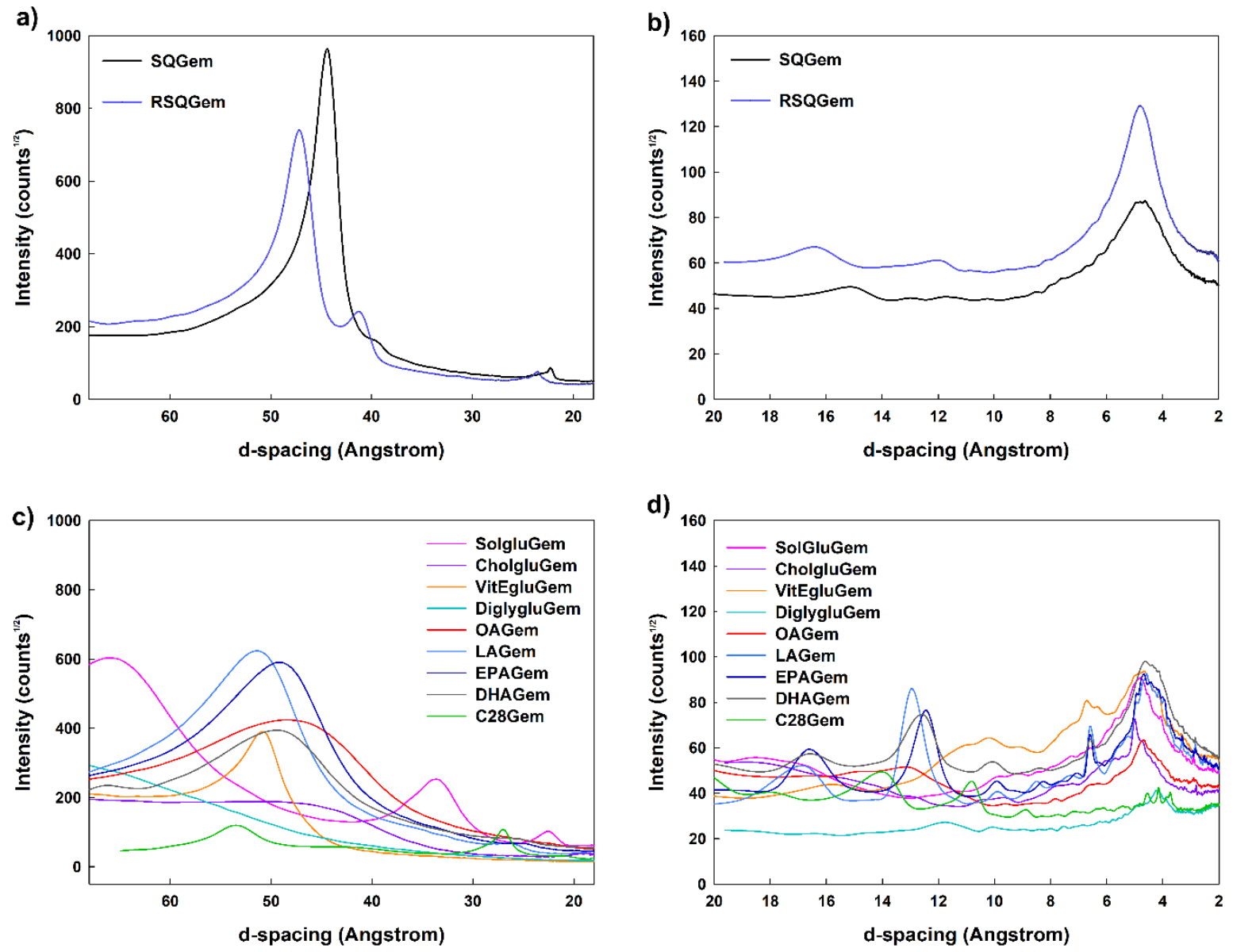

Figure 5. Powder X-ray pattern of gemcitabine prodrugs at (a,

c) low angles $\left(1.3<2 \theta_{\mathrm{Cu}}<5^{\circ}\right)$, and (b, d) high angles $\left(3.5<2 \theta_{\mathrm{Cu}}<50^{\circ}\right)$.

\section{Nanoparticle preparation and characterization}


Being the already described SQGem prodrug (Table 1, \#01) capable to spontaneously selfassemble as nanoparticles at a concentration of at least $1 \mathrm{mg} \cdot \mathrm{mL}^{-1}$ in water without addition of any surface active agent, whether the other synthetised prodrugs would display the same properties has been herein investigated. After having identified the best organic solvent for each prodrug (i.e., ethanol (EtOH), acetone (Ac) or tetrahydrofuran (THF)), the corresponding solution has been added dropwise into water under stirring. Among the 10 investigated conjugates four assembled spontaneously into stable nanoparticles at the desired concentration. On the contrary, despite tuning some experimental conditions (i.e., solvent nature, organic/aqueous phase ratio) the other lipid prodrugs, including LAGem, immediately precipitated upon the addition step or aggregated in the aqueous phase during/or rapidly after solvent removal.

Recently, however, the nanoprecipitation of a DMSO solution of LAGem conjugates has been reported as a strategy to formulate these conjugates into nanoparticles. ${ }^{57}$ Nevertheless, no details on the final concentration of the nanoassemblies have been provided. In addition, prior to in vitro and in vivo administration, these nanoassemblies were modified by the incorporation of PEGylated phospholipids, thus raising concerns about the stability of the initial LAGem nanoformulation.

Although nanoprecipitation was the method of choice for a suitable comparison with the SQGem prodrug, we also investigated other strategies such as the emulsion-evaporation ${ }^{58}$ (with SolgluGem, EPAGem and DHAGem) or the thin film hydration technique ${ }^{59}$ (with DiglygluGem and OAGem). However, the effort was vain and not any of these strategies allowed to form stable nanoassemblies satisfying our requirements (that is, pure water as aqueous phase and concentration of at least $1 \mathrm{mg} \cdot \mathrm{mL}^{-1}$ ).

These results clearly explained why previously described fatty-acid prodrugs of gemcitabine were administered only as organic solution in DMSO, ${ }^{60-62}$ or mixtures with surface active 
agents such as Pluronic, ${ }^{53}$ polyethylene glycol, ${ }^{57,63}$ Cremophor EL (i.e., polyoxyethylene castor oil $)^{64}$ or Tween $80 .^{31,65,66}$ In spite of their utilization also in clinically approved

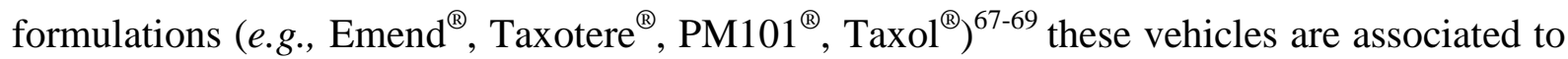
undesired toxicity, and could negatively affect the drug pharmacokinetics thus altering its efficacy. ${ }^{70-74}$ In this context, a successful attempt to develop a prodrug formulation in aqueous solvent would be extremely beneficial to face these drawbacks.

Formulated NPs displayed a mean diameter between 80 and $190 \mathrm{~nm}$, a narrow size distribution and a negative surface charge (Table 1). These NPs were stable in water over $72 \mathrm{~h}$ at room temperature (Figure 6a, Figure S23) while a slight size growth was immediately observed in complete cell culture medium likely due to the adsorption of the serum proteins at the NP surface (Figure 6b). The most important augmentation was recorded for the RSQGem NPs. Cryo-transmission electron microscopy (cryo-TEM) imaging of these NPs revealed that the RSQGem conjugates assembled into a dispersion of linear fibers (Figure 7b), while CholgluGem and VitEgluGem NPs (Figure 7d, e) formed elongated twisted filaments. As previously reported, the conjugation to an isoprenoid chain resulted, on the contrary, in the formation of $\quad$ spherical $\quad$ (Figure $\quad 7 \mathrm{a}, \quad \mathrm{c}){ }^{40,45}$ 
Table 1. Formulation conditions for the library of gemcitabine lipid conjugates and resulting nanoparticle characterization.

\begin{tabular}{|c|c|c|c|c|c|c|c|c|c|}
\hline \multicolumn{2}{|c|}{ Conjugate } & \multirow{2}{*}{$\begin{array}{l}\text { Nanoprecipitation } \\
\text { organic solvent }\end{array}$} & \multirow{2}{*}{$\begin{array}{l}\text { Organic } \\
\text { solvent/water } \\
\text { ratio }\end{array}$} & \multirow{2}{*}{$\begin{array}{l}\begin{array}{l}\mathrm{NP} \\
\text { concentration } \\
\left(\mathbf{m g}_{\mathrm{m}} \mathbf{m L}^{-1}\right)\end{array} \\
\mathbf{1}\end{array}$} & \multirow{2}{*}{$\begin{array}{l}\begin{array}{l}\text { Mean diameter } \\
(\mathbf{n m})\end{array} \\
\mathbf{8 9} \pm 8\end{array}$} & \multirow{2}{*}{$\begin{array}{l}\text { Polydispersity index } \\
\text { (PdI) } \\
0.09 \pm 0.03\end{array}$} & \multirow{2}{*}{$\begin{array}{l}\text { Zeta } \\
\text { Potential } \\
(\mathrm{mV})\end{array}$} & \multirow{2}{*}{$\begin{array}{l}\begin{array}{l}\text { Drug } \\
\text { loading }^{b} \\
\text { (wt \%) }\end{array} \\
\mathbf{4 0 . 7}\end{array}$} & \multirow{2}{*}{$\begin{array}{l}\text { HLB }^{c} \\
7.41\end{array}$} \\
\hline$\# 01$ & SQGem & & & & & & & & \\
\hline \#02 & RSQGem & EtOH & $1: 2$ & 1 & $121 \pm 3$ & $0.03 \pm 0.02$ & $-11 \pm 1$ & 40.1 & 7.30 \\
\hline$\# 03$ & SolgluGem & THF & $1: 2$ & 1 & $157 \pm 32$ & $0.07 \pm 0.02$ & $-53 \pm 8$ & 26.6 & 6.00 \\
\hline$\# 04$ & CholgluGem & Ac & $1: 2$ & 1 & $112 \pm 15$ & $0.23 \pm 0.02$ & $-31 \pm 6$ & 35.2 & 7.95 \\
\hline \#05 & VitEgluGem & THF & $1: 4$ & 1 & $146 \pm 17$ & $0.11 \pm 0.03$ & $-36 \pm 17$ & 33.3 & 8.49 \\
\hline \#06 & DiglygluGem & THF & $1: 2$ & 1 & Aggregation & - & - & 28.4 & 8.89 \\
\hline \multirow{3}{*}{ \#07 } & \multirow{3}{*}{ OAGem } & EtOH & $1: 2$ & 1 & Aggregation & - & - & \multirow{3}{*}{49.9} & \multirow{3}{*}{9.07} \\
\hline & & Ac & $1: 2$ & 1 & Aggregation & - & - & & \\
\hline & & THF & $1: 2$ & 1 & Aggregation & - & - & & \\
\hline \multirow{3}{*}{$\# 08$} & \multirow{3}{*}{ LAGem } & EtOH & $1: 2$ & 1 & Aggregation & - & - & \multirow{3}{*}{50.1} & \multirow{3}{*}{9.10} \\
\hline & & Ac & $1: 2$ & 1 & Aggregation & - & - & & \\
\hline & & THF & $1: 2$ & 1 & Aggregation & - & - & & \\
\hline \multirow{3}{*}{$\# 09$} & \multirow{3}{*}{ EPAGem } & EtOH & $1: 2$ & 1 & Aggregation & - & - & \multirow{3}{*}{48.1} & \multirow{3}{*}{8.74} \\
\hline & & Ac & $1: 2$ & 1 & Aggregation & - & - & & \\
\hline & & THF & $1: 2$ & 1 & Aggregation & - & - & & \\
\hline \multirow{3}{*}{$\# 10$} & \multirow{3}{*}{ DHAGem } & EtOH & $1: 2$ & 1 & Aggregation & - & - & \multirow{3}{*}{45.9} & \multirow{3}{*}{8.34} \\
\hline & & Ac & $1: 2$ & 1 & Aggregation & - & - & & \\
\hline & & THF & $1: 2$ & 1 & Aggregation & - & - & & \\
\hline \#11 & C28Gem & THF & $1: 2$ & 1 & Aggregation & - & - & 39.3 & 7.14 \\
\hline
\end{tabular}


a)

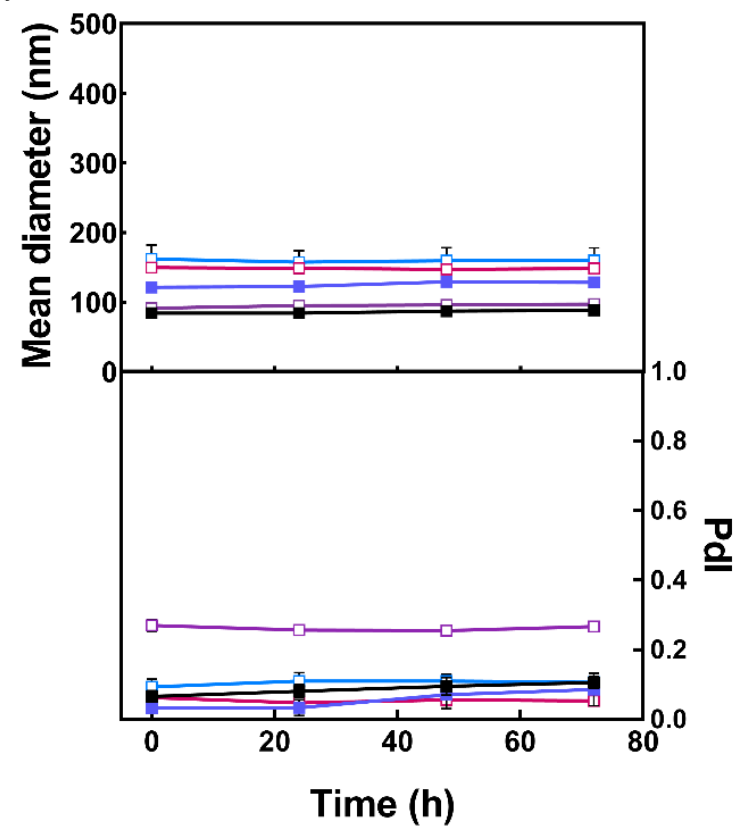

b)

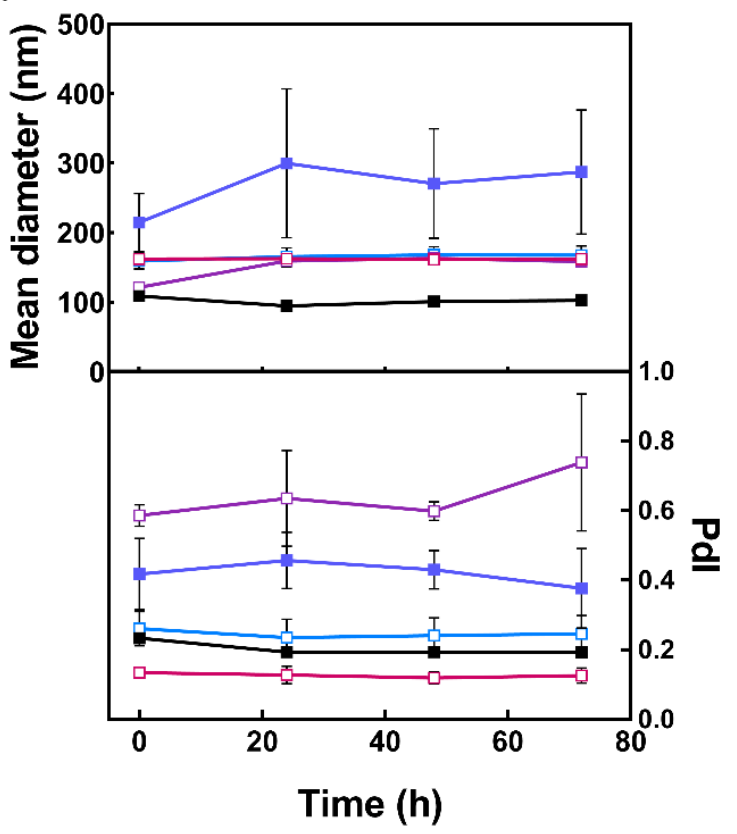

Figure 6. Evolution of NP mean diameter and polydispersity index (PdI) after incubation in (a) water at room temperature and (b) L-15 cell culture medium supplemented with $10 \%$ FBS at $37^{\circ} \mathrm{C}$. Values represents mean \pm standard deviation $(n=3)$.
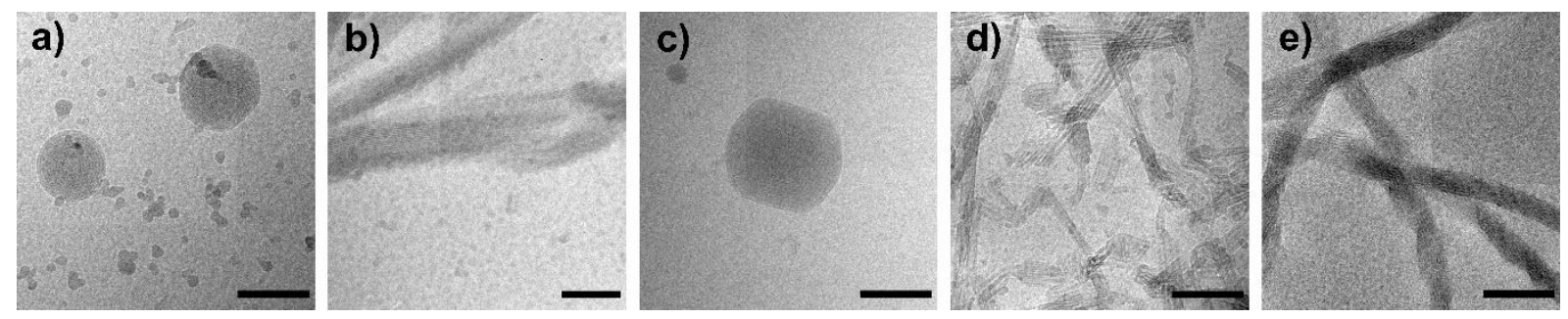

Figure 7. Representative cryoTEM images of (a) SQGem, (b) RSQGem, (c) SolgluGem, (d) CholgluGem and (e) VitEgluGem nanoparticles. Scale bar $=100 \mathrm{~nm}$.

X-ray diffraction pattern of the various prodrugs (Figure 5, Table S1) did not highlight a correlation with their capacity (or not) to self-assemble into stable nanoparticles. Thus, we investigated whether another physico-chemical parameter, namely their hydrophilic-lipophilic 
balance (HLB), could play a key role. The HLB values, calculated according to the Griffin method, ${ }^{75}$ ranged from 6.00 for the SolgluGem to 9.10 for the LAGem (Table 1) and a trend has emerged that, for values above 8.34 , the lipid conjugates were not able to spontaneously self-assemble as nanoparticles (Figure 8a). Nevertheless, due to the limited number of prodrugs included in this first analysis, it was hard to extrapolate a general behavior. Accordingly, to better understand whether the HLB value could be an indicator of the ability of a conjugate to self-assemble into NPs, we introduced in this study several prodrugs already synthetised in our group over the past 15 years. We first focused on the gemcitabine prodrugs only (Figure 8b) and then we also included the whole library of available nucleoside analogue derivatives, which successfully self-organized into nanoparticles by nanoprecipitation in pure water, at the minimum concentration of $1 \mathrm{mg} \cdot \mathrm{mL}^{-1}$ (Figure $8 \mathrm{c}$ ). ${ }^{45,76-79}$ In both cases, we observed a significant difference between the HLB values median. In the last group, which included the larger number of prodrugs, these medians were of 7.45 and 9.07 for the prodrugs which self-assembled or not, respectively (Figure 8c). 

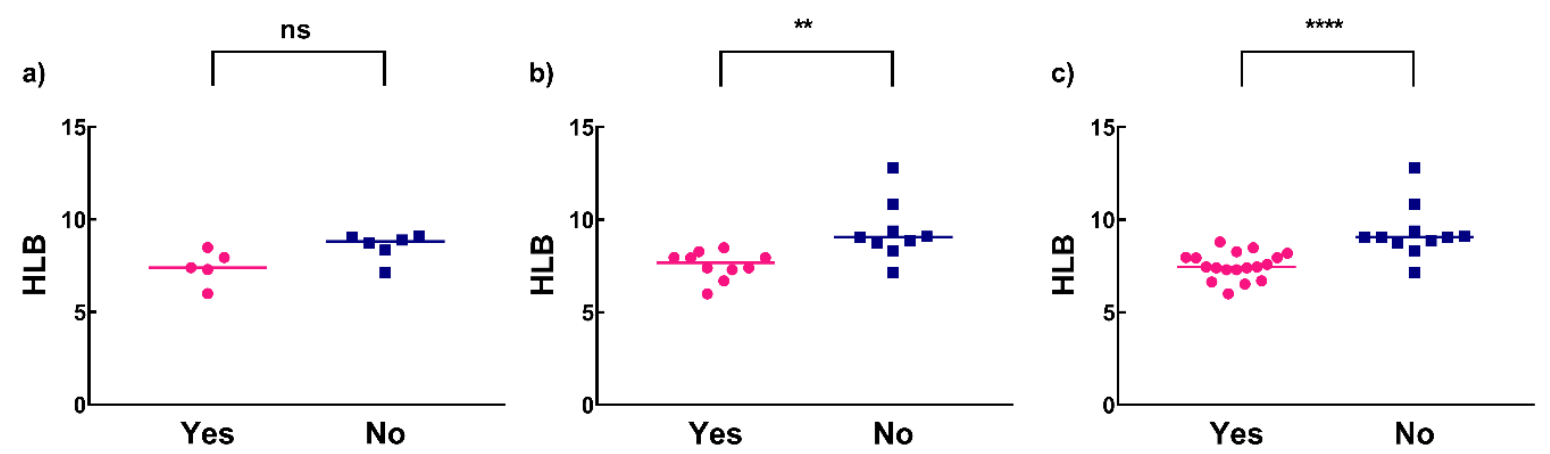

Figure 8. Comparison of HLB values between prodrugs able (Yes) or not (No) to selfassemble in form of nanoparticles in pure water using the nanoprecipitation technique. (Scatter plot, median; ns: non-significant, ${ }^{*} p<0.0021$, **** $p<0.0001$ Mann-Whitney's test). (a) lipid prodrugs synthetised by coupling the Gem to the lipid moieties in Figure 1. (b) pool of all the gemcitabine lipid prodrugs previously synthetised by us (including (a)); (c) pool of all nucleoside lipid prodrugs previously synthetised by us (including (b)). Structures and HLB of each prodrug used in this figure are reported in Table S2.

Overall, these results confirmed that an HLB below 8.34 (that is, the lowest value in the group that does not form nanoparticles) could be a good indicator of the category to which the prodrug belongs. In agreement with this hypothesis is the observation that other gemcitabine prodrugs described in literature (i.e., research groups other than ours) and reported as unable to form stable NPs, had an HLB above this critical value (Table S3). ${ }^{34,53}$

The identification of few exceptions (i.e., C28Gem (\#11) and the VitEgluGem (\#05)) suggests that the HLB value although predictive is, however, not sufficient alone to determine whether a conjugate is able or not to form nanoassemblies. Indeed, multiple other parameters such as $\pi$-stacking and hydrogen bonding could affect the self-organization process, as previously observed with various nucleolipids. ${ }^{80-83}$

A close regard to the lipid chain of the prodrugs that successfully assembled as nanoparticles revealed a shared isoprene-like structural unit showing branched methyl groups on the main 
carbon backbone. The latter are likely to hinder the establishment of too strong hydrophobic interactions between the prodrug molecules, which could spatially organize thus leading to the observed NPs. On the contrary, during the nanoprecipitation a close packing of the other prodrugs probably occurred with the formation of solid insoluble aggregates.

\section{In vitro studies}

The cytotoxicity of the formulated NPs was investigated on the MDA-MB-23 cells, a wellknown model of aggressive breast cancer characterized by a high lipoprotein receptor activity. As also previously observed, the SQGem NPs showed a higher cytotoxicity comparatively to the free drug (IC50 of $1.3 \mu \mathrm{M} v s 2.7 \mu \mathrm{M}$ ). ${ }^{79,84}$ On the contrary, the other NP formulations displayed only mild cytotoxicity even in the micromolar range (Figure 9). The slower gemcitabine release from the SolgluGem NPs, herein selected as an example of a nanoformulation with a low activity comparatively to the SQGem NPs, is probably responsible for the limited biological efficacy (Figure S24). These results showed that, in spite of the capacity of these prodrugs to assemble as nanoparticles, the replacement of the squalene chain with other lipid moieties seemed to be, at least in vitro, therapeutically irrelevant.

Yet, in vivo, these nanoparticles might show a different behavior. It is well known that lipophilic drugs can bind to lipoproteins, so it is possible to speculate that the HLB value of the prodrugs would affect their distribution profile among different plasma components (lipoproteins, proteins) and consequently their therapeutic activity. ${ }^{85}$

However, as previously observed with various molecular species, ${ }^{86,87}$ a prediction of the fate of gemcitabine prodrugs based on hydrophobicity only could be rather reductive. In addition to the HLB, other parameters such as binding constants, relative abundance of plasma 
components, as well as the colloidal stability of the nanocarriers into the blood circulation, should be studied in depth in order to extrapolate a broad structure-activity relationship.

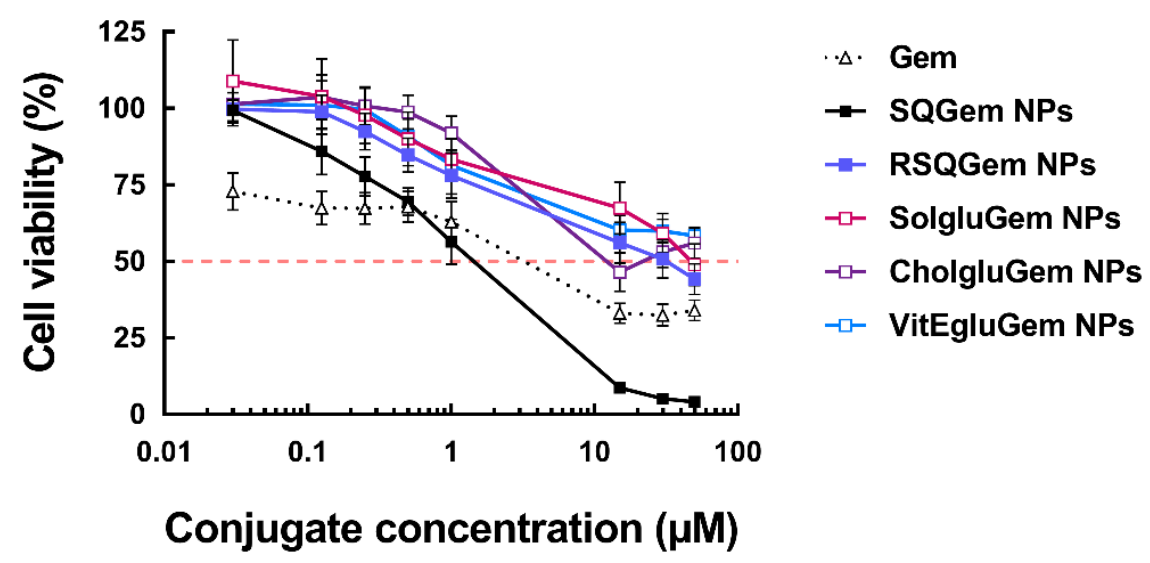

Figure 9. Cell viability of MDA-MB-231 cells after incubation with increasing concentrations of free Gem, SQGem, RSQGem, SolgluGem, CholgluGem and VitEgluGem nanoparticles. Values represent mean \pm standard deviation $(n \geq 3)$.

\section{Conclusions}

By using a small library of amphiphilic lipid prodrugs of gemcitabine, we investigated how the nature of the lipid moieties could affect the capacity (or not) to self-assemble as nanoparticles in pure water without the addition of any surfactant. The HLB value was retained as a valuable predictive parameter. Indeed, although with some exceptions, conjugates having an HLB over 8.34 could not be formulated as stable nanoparticles. The presence of lateral methyl on the hydrophobic chain could also be a key parameter in the ability of the bioconjugates to self-assemble. However, the nanoparticulate form was not the guaranty, at least in vitro, of a relevant biological activity since the NPs other than SQGem displayed only moderated cytotoxicity comparatively to the free drug and to the SQGem NPs, which once again confirmed their efficacy. Overall, these findings might help the design of 
optimal conjugates which, thanks to their self-assembling ability, could be formulated as nanoparticles without the need of using potentially toxic vehicles.

\section{Experimental Procedures}

\section{Materials}

Regular analytical grade and deuterated solvents were supplied by Carlo Erba (France) and Eurisotop (France), respectively. Tetrahydrofuran (THF) was distilled from sodium/benzophenone ketyl. Toluene, pyridine, dimethylformamide (DMF), and dichloromethane (DCM) were distilled from calcium hydride, under a nitrogen atmosphere. All reactions involving air- or water-sensitive compounds were routinely conducted in glassware which was flame-dried under a positive pressure of nitrogen. Gemcitabine (Gem) hydrochloride, gemcitabine base, solanesol (Sol), eicosapentaenoic acid (EPA), docosahexaenoic acid (DHA) and 4-dimethylaminopyridine (DMAP) were purchased from Carbosynth Ltd (UK). Squalene (SQ), cholesterol (Chol), vitamin E (VitE), oleic acid (OA), linoleic acid (LA), octacosanoic acid (C28), glutaric anhydride, benzotriazole-1-yl-oxy-trispyrrolidino-phosphonium hexafluorophosphate (pyBOP), ethyl chloroformate, $\mathrm{N}, \mathrm{N}$ diisopropylethylamine (diPEA), triethylamine, tert-butyldimethylsilyl chloride, imidazole and tetra- $n$-butylammonium fluoride (TBAF) were purchased from Sigma-Aldrich (France). Chemicals obtained from commercial suppliers were used without further purification.

\section{Analytical techniques}

Infrared (IR) spectra were obtained as solid or neat liquid on a Fourier Transform Bruker Vector 22 spectrometer. Only significant absorptions are listed. Optical rotations were measured on a PerkinElmer 241 Polarimeter at $589 \mathrm{~nm}$. The ${ }^{1} \mathrm{H}$ and ${ }^{13} \mathrm{C}$ NMR spectra were recorded on Bruker Avance $300\left(300 \mathrm{MHz}\right.$ and $75 \mathrm{MHz}$ for ${ }^{1} \mathrm{H}$ and ${ }^{13} \mathrm{C}$, respectively) or Bruker Avance 400 (400 MHz and $100 \mathrm{MHz}$ for ${ }^{1} \mathrm{H}$ and ${ }^{13} \mathrm{C}$, respectively) spectrometers. 
Recognition of methyl, methylene, methine, and quaternary carbon nuclei in ${ }^{13} \mathrm{C}$ NMR spectra rests on the $J$-modulated spin-echo sequence. Mass spectra (MS) and high-resolution mass spectra (HRMS) were recorded using electrospray ionization (ESI) on an LTQ-Velos Pro mass spectrometer (ThermoScientific, Germany). Analytical thin-layer chromatography was performed on Merck silica gel $60 \mathrm{~F}_{254}$ glass precoated plates $(0.25 \mathrm{~mm}$ layer $)$. Column chromatography was performed on Merck silica gel 60 (230-400 mesh ASTM).

\section{Hydrophilic Lipophilic Balance calculations}

The hydrophilic-lipophilic balance (HLB) values of the lipid prodrugs were determined using MarvinSketch Version 21.3 software (ChemAxon, 2021). The HLB was calculated with the HLB Predictor plug-in using the Griffin empirical formula: HLB $=20(1-\mathrm{S} / \mathrm{A})$, where S and A correspond to the saponification and the acid numbers of the molecule, respectively. ${ }^{75}$

\section{DRX analysis}

X-Ray diffraction (XRD) experiments were carried out on a high-resolution $\theta-\theta$ powder diffractometer (D8 Advance, Bruker AXS, Germany) equipped with the LynxEye XE-T detector (1D mode), dynamic beam optimization module (DBO, combining the use of variable slits, automatic anti-scatter screen position and controlled detector opening) and a $\mathrm{Cu}$ radiation $\left(\mathrm{K}_{\alpha 1}=1.5406 \AA\right.$ and $\left.\mathrm{K}_{\alpha 2}=1.5445 \AA\right)$. The former drastically reduced the diffusion signal at very low angles $\left(2 \theta_{\mathrm{Cu}}<10^{\circ}\right)$ and combined with a careful control of the sample height, it allowed to obtain reproducible qualitative information on samples exhibiting $d$ spacing up to about $68 \AA$. All prodrugs were dissolved into an optimal organic solvent (that is, DCM (SQGem, SolgluGem VitEgluGem, DiglygluGem, LAGem, EPAGem DHAGem); EtOH (RSQGem and OAGem) or THF (CholgluGem and C28Gem)) at a concentration of either $12.5 \mathrm{mg} \cdot \mathrm{mL}^{-1}$ or $25 \mathrm{mg} \cdot \mathrm{mL}^{-1}$ depending on their solubility. $2.5 \mathrm{mg}$ were deposited onto 
zero background silicon wafers (24 mm diameter, Siltronix, France). Solvent was evaporated at room temperature prior to analysis, resulting in a thin film of the lipid prodrugs onto the wafer surface. Samples were analyzed at room temperature with a 10 -rpm rotation, $2.5^{\circ}$ Sollers slits and a sample illumination surface fixed at $10 \mathrm{~mm}$ (variable slits mode). XRD patterns were acquired at two different theta ranges: ( $i$ ) from $1.3-5^{\circ}$ ( $2 \theta$ range) with a step size of $0.03^{\circ}$ and $5 \mathrm{~s}$ per step ( $\mathrm{n}=3$ per prodrug), an anti-scatter screen fixed at $0.5 \mathrm{~mm}$ from the surface of the film, a limited detector opening (PSD $\left.=1^{\circ}\right)$ and (ii) from $3.5-50^{\circ}(2 \theta$ range) with an anti-scatter screen position automatically optimized by the software, a full detector opening, with a step size of $0.03^{\circ}$ and 4 s per step ( $n=1$ per prodrug).

\section{NP formulation and characterization}

Lipid prodrug nanoparticles were prepared according to the nanoprecipitation methodology (Table 1). ${ }^{38,88}$ Briefly, prodrugs were dissolved at $2 \mathrm{mg} \cdot \mathrm{mL}^{-1}$ in a water-miscible organic solvent (EtOH for SQGem, RSQGem, DiglygluGem, OAGem, LAGem, EPAGem; acetone for CholgluGem, OAGem, LAGem, EPAGem, DHAGem; THF for SolgluGem, VitEgluGem, DiglygluGem, OAGem, LAGem, EPAGem, DHAGem, C28Gem) (Table 1). $500 \mu \mathrm{L}$ of the resulting solution were then added dropwise to a stirred solution of MilliQ water at solvent to water ratio of 1:2 v/v (SQGem, RSQGem, SolgluGem, CholgluGem, DiglygluGem, OAGem, LAGem, EPAGem, DHAGem and C28Gem) or 1:4 v/v (VitEgluGem). After removal of the organic solvent under reduced pressure, an aqueous dispersion of NPs at a final concentration of $1 \mathrm{mg} \cdot \mathrm{mL}^{-1}$ was (or not) obtained. In order to reach this value, VitEgluGem NPs were concentrated under vacuum by removal of $1 \mathrm{~mL}$ of water.

When NPs were obtained, mean diameter and size distribution were measured by dynamic light scattering with a Nano ZS from Malvern $\left(173^{\circ}\right.$ scattering angle) at $25{ }^{\circ} \mathrm{C}$. The measurements were performed after NP dilution at $0.1 \mathrm{mg} \cdot \mathrm{mL}^{-1}$ in Milli-Q water. The NP 
surface charge was investigated by $\zeta$-potential measurement at $25{ }^{\circ} \mathrm{C}$ after dilution at 0.05 mg. $\mathrm{mL}^{-1}$ in $1 \mathrm{mM} \mathrm{NaCl}$ solution applying the Smoluchowski equation and using the same apparatus. Measurements were carried out at least in triplicate. Colloidal stability has been assessed by measuring NP mean diameter and size distribution over a period of $72 \mathrm{~h}$. NPs have been diluted to $0.1 \mathrm{mg} \cdot \mathrm{mL}^{-1}$ in Milli-Q water or in complete cell culture medium (L-15 Leibovitz supplemented with $10 \%$ of fetal bovine serum) and incubated at $4{ }^{\circ} \mathrm{C}$ and room temperature or at $37^{\circ} \mathrm{C}$, respectively.

\section{Cryo-transmission electron microscopy}

NP morphology has been examined by cryo-transmission electron microscopy (cryoTEM).

Briefly, $5 \mu \mathrm{L}$ of NP dispersion at a concentration of $1 \mathrm{mg} \cdot \mathrm{mL}^{-1}$ for SQGem, RSQGem, SolgluGem and VitEgluGem NPs and of $0.5 \mathrm{mg} \cdot \mathrm{mL}^{-1}$ for CholgluGem NPs, were deposited onto a 200-mesh holey carbon copper grid (TedPella Inc.) and flash-frozen in liquid ethane cooled down at liquid nitrogen temperature. Cryo-TEM images were acquired on a JEOL 2200FS energy-filtered $(20 \mathrm{eV})$ field emission gun electron microscope operating at $200 \mathrm{kV}$ using a Gatan ssCCD $2048 \times 2048$ pixels.

\section{Gemcitabine release}

The release of gemcitabine from SQGem and SolgluGem NPs was investigated in the presence of serum according to a previously described procedure with slight modifications. ${ }^{89,90}$ Practically, nanoparticles $(200 \mu \mathrm{L})$ were incubated with $800 \mu \mathrm{L}$ of human serum (Sigma Aldrich, France) supplemented with $570 \mathrm{mg} \cdot \mathrm{mL}^{-1}$ tetrahydrouridine (THU) at $37^{\circ} \mathrm{C}$ with gentle shaking. At predetermined time points $(\mathrm{t}=0,1,2,4,8$ and $24 \mathrm{~h})$, aliquots $(100 \mu \mathrm{L})$ were withdrawn and spiked with $10 \mu \mathrm{L}$ of the internal standard solution (theophylline, $10 \mu \mathrm{M}$ in $\mathrm{mQ}$ water) before addition of $1 \mathrm{~mL}$ of a mixture of 
acetonitrile:methanol (90:10, v/v) and ultracentrifuged $\left(20 \mathrm{~min}, 4{ }^{\circ} \mathrm{C}, 15000 \mathrm{~g}\right)$. The supernatant was then evaporated to dryness at $30^{\circ} \mathrm{C}$ under a nitrogen flow. The released drug was quantified by reverse-phase HPLC. The chromatographic system consisted of a Waters 1525 Binary LC pump, a Waters 2707 Autosampler, a Waters 2998 programmable photodiode-array detector (Waters, Milford, MA 01757, USA) and a C18 Uptisphere column $\left(3 \mu \mathrm{m}, 150 \times 4.6 \mathrm{~mm}\right.$; Interchim). The HPLC column was maintained at $30{ }^{\circ} \mathrm{C}$. Detection was monitored at $270 \mathrm{~nm}$. The HPLC mobile phase consisted of a mixture of methanol/water with $0.05 \mathrm{M}$ sodium acetate ( $\mathrm{pH}$ 5; solvent A: 5/95, v/v; solvent B 97/3, v/v). The residues were dissolved in solvent A $(100 \mu \mathrm{L})$. Elution was performed at a flow rate of $0.8 \mathrm{~mL} / \mathrm{min}$ isocratically for $11 \mathrm{~min}$ with solvent A followed by a linear gradient (1 minute) to $75 \%$ solvent A. After a 6-min hold, a 100\% solvent B was achieved by a linear gradient (1 minute) and kept for 10 minutes. After a linear gradient $(1 \mathrm{~min})$ to $100 \%$ solvent $\mathrm{A}$, the system was held for $5 \mathrm{~min}$ for equilibration back to initial status.

\section{Cell culture}

Human breast cancer cells MDA-MB-231 were obtained from the American Type Culture Collection and cultured as recommended. Briefly, cells were maintained in Leibovitz's L15 medium supplemented with $15 \%$ fetal bovine serum, $2 \mathrm{mg} \cdot \mathrm{mL}^{-1}$ sodium bicarbonate, 50 U.mL ${ }^{-1}$ penicillin, $50 \mu \mathrm{g} \cdot \mathrm{mL}^{-1}$ streptomycin, and $2 \mathrm{mM}$ glutamine. Cells were maintained in a humid atmosphere at $37{ }^{\circ} \mathrm{C}$ with $5 \% \mathrm{CO}_{2}$.

\section{Cytotoxicity evaluation}

MDA-MB-231 were seeded in 96-wells plates (5000 cells per well in $100 \mu \mathrm{L}$ of complete culture medium) $24 \mathrm{~h}$ prior to exposure of a series of concentration of NPs or free Gem for $72 \mathrm{~h}$. Cell viability was determined based on the mitochondrial dehydrogenase activity using 
the 3-[4, 5-dimethylthiazol-2-yl]-3,5-diphenyl tetrazolium bromide (MTT) test. At the end of the incubation period, $20 \mu \mathrm{L}$ of a MTT solution $\left(5 \mathrm{mg} \cdot \mathrm{mL}^{-1}\right.$ in PBS) were added to each well and plates were incubated for $2 \mathrm{~h}$ at $37^{\circ} \mathrm{C}$. Subsequently, the medium was gently removed, and the formazan crystals were dissolved in dimethylsulfoxide (200 $\mu \mathrm{L}$ per well). Absorbance of the dye solution was measured at $570 \mathrm{~nm}$ using a microplate reader (LAB System Original Multiscan MS). The percentage of viable cells in each well was calculated as the absorbance ratio between treated and non-treated control cells. All experiments were set up at least in triplicate to determine means and standard deviation (SD).

\section{Associated content}

\section{Supporting Information}

The Supporting Information is available free of charge on the ACS Publications website at DOI: $\mathrm{xxxx}$

Detailed synthesis of gemcitabine lipid prodrugs. Supporting figures: NMR spectra; zeta potential of nanoparticles over time; gemcitabine release from SQGem and SolgluGem NPs.

Supporting tables: $d$-spacing of gemcitabine lipid prodrugs; list of the lipid prodrugs investigated in Figure 8; list of the lipid prodrugs described in literature which do not form NP by nanoprecipitation. 


\section{Author information}

\section{Corresponding author}

*E-mail: simona.mura@universite-paris-saclay.fr

\section{ORCID}

Eléonore Coppens 0000-0002-0566-2172

Didier Desmaële 0000-0003-4013-4655

Julie Mougin 0000-0002-2118-860X

Sandrine Tusseau-Nenez 0000-0001-6683-2385

Patrick Couvreur 0000-0001-7961-5443

Simona Mura 0000-0003-0310-8052

\section{Notes}

Patrick Couvreur is co-founder of Squal Pharma https://squalpharma.com/.

\section{Acknowledgments}

This work was supported by the French Ministry of Research, the Fondation pour la Recherche Médicale (Application FDT202001010776) and the Ligue contre le cancer (Subvention Recherche HB2019-22 and NH2020-32). The CNRS is also warmly acknowledged for financial support. We acknowledge Dr Audrey Solgadi and Bastien Prost (UMS-IPSIT-Plateforme SAMM, France) for mass spectrometry analysis, the Electron 
Microscopy Facility of the Multimodal Imaging Centre at Institut Curie, Orsay for providing access to the electron microscope and Dr Sylvain Trépout (Institut Curie, France) for the cryoTEM images. We would also like to thank Miss Stéphanie Denis and Miss Clélia Mathieu (Institut Galien Paris-Saclay, France) for their support in cell culture experiments, and Miss Maëlle Mouzard for her help in the chemical synthesis as part of her L2 internship. 


\section{References}

(1) Di, L., Fish, P. V., and Mano, T. (2012) Bridging solubility between drug discovery and development. Drug Discov. Today 17, 486-495.

(2) Rautio, J., Meanwell, N. A., Di, L., and Hageman, M. J. (2018) The expanding role of prodrugs in contemporary drug design and development. Nat. Rev. Drug Discov. 17, 559-587.

(3) Stella, V. J., and Nti-Addae, K. W. (2007) Prodrug strategies to overcome poor water solubility. Adv. Drug Deliv. Rev. 59, 677-694.

(4) Xu, Y., Mu, J., Xu, Z., Zhong, H., Chen, Z., Ni, Q., Liang, X.-J., and Guo, S. (2020) Modular Acid-Activatable Acetone-Based Ketal-Linked Nanomedicine by Dexamethasone Prodrugs for Enhanced Anti-Rheumatoid Arthritis with Low Side Effects. Nano Lett. 20, 2558-2568.

(5) Mura, S., Duc Trung, B., Couvreur, P., and Nicolas, J. (2015) Lipid prodrug nanocarriers in cancer therapy. J. Control. Release 208, 25-41.

(6) Fattahi, N., Shahbazi, M.-A., Maleki, A., Hamidi, M., Ramazani, A., and Santos, H. A. (2020) Emerging insights on drug delivery by fatty acid mediated synthesis of lipophilic prodrugs as novel nanomedicines. J. Control. Release 326, 556-598.

(7) Wang, Y., Cheetham, A. G., Angacian, G., Su, H., Xie, L., and Cui, H. (2017) Peptide-drug conjugates as effective prodrug strategies for targeted delivery. Adv. Drug Deliv. Rev. 110-111, 112-126.

(8) Delplace, V., Couvreur, P., and Nicolas, J. (2014) Recent trends in the design of anticancer polymer prodrug nanocarriers. Polym. Chem. 5, 1529-1544.

(9) Seidi, F., Jenjob, R., and Crespy, D. (2018) Designing Smart Polymer Conjugates for Controlled Release of Payloads. Chem. Rev. 118, 3965-4036.

(10) Zhong, H., Mu, J., Du, Y., Xu, Z., Xu, Y., Yu, N., Zhang, S., and Guo, S. (2020) AcidTriggered Release of Native Gemcitabine Conjugated in Polyketal Nanoparticles for Enhanced Anticancer Therapy. Biomacromolecules 21, 803-814.

(11) Stella, V. J. (2004) Prodrugs as therapeutics. Expert Opin. Ther. Pat. 14, 277-280.

(12) Rautio, J., Kumpulainen, H., Heimbach, T., Oliyai, R., Oh, D., Järvinen, T., and Savolainen, J. (2008) Prodrugs: design and clinical applications. Nat. Rev. Drug Discov. 7, 255-270.

(13) Huttunen, K. M., Raunio, H., and Rautio, J. (2011) Prodrugs-from Serendipity to Rational Design. Pharmacol. Rev. 63, 750. 
(14) Rautio, J., Laine, K., Gynther, M., and Savolainen, J. (2008) Prodrug approaches for CNS delivery. AAPS J. 10, 92-102.

(15) Porter, C. J. H., Trevaskis, N. L., and Charman, W. N. (2007) Lipids and lipid-based formulations: optimizing the oral delivery of lipophilic drugs. Nat. Rev. Drug Discov. 6, 231248.

(16) Irby, D., Du, C., and Li, F. (2017) Lipid-Drug Conjugate for Enhancing Drug Delivery. Mol. Pharm. 14, 1325-1338.

(17) Dahan, A., Markovic, M., Aponick, A., Zimmermann, E. M., and Ben-Shabat, S. (2019) The prospects of lipidic prodrugs: an old approach with an emerging future. Future Med. Chem. 11, 2563-2571.

(18) Han, S., Hu, L., Gracia, Quach, T., Simpson, J. S., Edwards, G. A., Trevaskis, N. L., and Porter, C. J. (2016) Lymphatic Transport and Lymphocyte Targeting of a Triglyceride Mimetic Prodrug Is Enhanced in a Large Animal Model: Studies in Greyhound Dogs. Mol. Pharm. 13, 3351-3361.

(19) Radwan, A. A., and Alanazi, F. K. (2014) Targeting cancer using cholesterol conjugates. Saudi Pharm. J. 22, 3-16.

(20) Wang, H., Feng, Z., Wu, D., Fritzsching, K. J., Rigney, M., Zhou, J., Jiang, Y., Schmidt-Rohr, K., and Xu, B. (2016) Enzyme-Regulated Supramolecular Assemblies of Cholesterol Conjugates against Drug-Resistant Ovarian Cancer Cells. J. Am. Chem. Soc. 138, 10758-10761.

(21) Sparreboom, A., Wolff, A. C., Verweij, J., Zabelina, Y., van Zomeren, D. M., McIntire, G. L., Swindell, C. S., Donehower, R. C., and Baker, S. D. (2003) Disposition of docosahexaenoic acid-paclitaxel, a novel taxane, in blood: in vitro and clinical pharmacokinetic studies. Clin. Cancer Res. 9, 151-9.

(22) Luo, C., Sun, J., Liu, D., Sun, B., Miao, L., Musetti, S., Li, J., Han, X., Du, Y., Li, L., et al. (2016) Self-Assembled Redox Dual-Responsive Prodrug-Nanosystem Formed by Single Thioether-Bridged Paclitaxel-Fatty Acid Conjugate for Cancer Chemotherapy. Nano Lett. 16, 5401-5408.

(23) Arpicco, S., Lerda, C., Dalla Pozza, E., Costanzo, C., Tsapis, N., Stella, B., Donadelli, M., Dando, I., Fattal, E., Cattel, L., et al. (2013) Hyaluronic acid-coated liposomes for active targeting of gemcitabine. Eur. J. Pharm. Biopharm. 85, 373-380.

(24) May, J. P., Undzys, E., Roy, A., and Li, S.-D. (2016) Synthesis of a Gemcitabine Prodrug for Remote Loading into Liposomes and Improved Therapeutic Effect. Bioconjug. Chem. 27, 226-237. 
(25) Li, P. W., Luo, S., Xiao, L. Y., Tian, B. L., Wang, L., Zhang, Z. R., and Zeng, Y. C. (2019) A novel gemcitabine derivative-loaded liposome with great pancreas-targeting ability. Acta Pharmacol. Sin. 40, 1448-1456.

(26) Tretiakova, D. S., Khaidukov, S. V., Babayants, A. A., Frolova, I. S., Shcheglovitova, O. N., Onishchenko, N. R., and Vodovozova, E. L. (2020) Lipophilic Prodrug of Methotrexate in the Membrane of Liposomes Promotes Their Uptake by Human Blood Phagocytes. Acta Naturae 12, 99-109.

(27) Tretiakova, D., Svirshchevskaya, E., Onishchenko, N., Alekseeva, A., Boldyrev, I., Kamyshinsky, R., Natykan, A., Lokhmotov, A., Arantseva, D., Shobolov, D., et al. (2020) Liposomal Formulation of a Melphalan Lipophilic Prodrug: Studies of Acute Toxicity, Tolerability, and Antitumor Efficacy. Curr. Drug Deliv. 17, 312-323.

(28) Stevens, P. J., Sekido, M., and Lee, R. J. (2004) Synthesis and evaluation of a hematoporphyrin derivative in a folate receptor-targeted solid-lipid nanoparticle formulation. Anticancer Res. 24, 161-5.

(29) Wang, C., Zheng, Y., Sand Oval, M. A., Valdes, S. A., Chen, Z., Lansakara, P. D., Du, M., Shi, Y., and Cui, Z. (2017) Oral 4-(N)-stearoyl gemcitabine nanoparticles inhibit tumor growth in mouse models. Oncotarget 8, 89876-89886.

(30) Stella, B., Peira, E., Dianzani, C., Gallarate, M., Battaglia, L., Gigliotti, C. L., Boggio, E., Dianzani, U., and Dosio, F. (2018) Development and Characterization of Solid Lipid Nanoparticles Loaded with a Highly Active Doxorubicin Derivative. Nanomaterials (Basel) 8,110 .

(31) Valdes, S. A., Alzhrani, R. F., Rodriguez, A., Lansakara-P, D. S. P., Thakkar, S. G., and Cui, Z. (2019) A solid lipid nanoparticle formulation of 4-(N)-docosahexaenoyl 2', 2'difluorodeoxycytidine with increased solubility, stability, and antitumor activity. Int. J. Pharm. 570, 118609.

(32) Zhu, S., Wonganan, P., Lansakara, P. D., O'Mary, H. L., Li, Y., and Cui, Z. (2013) The effect of the acid-sensitivity of 4-(N)-stearoyl gemcitabine-loaded micelles on drug resistance caused by RRM1 overexpression. Biomaterials 34, 2327-39.

(33) Daman, Z., Ostad, S., Amini, M., and Gilani, K. (2014) Preparation, optimization and in vitro characterization of stearoyl-gemcitabine polymeric micelles: A comparison with its self-assembled nanoparticles. Int. J. Pharm. 468, 142-151.

(34) Kakwere, H., Ingham, E. S., Tumbale, S. K., and Ferrara, K. W. (2020) Gemcitabineretinoid prodrug loaded nanoparticles display in vitro antitumor efficacy towards drugresilient human PANC-1 pancreatic cancer cells. Mater. Sci. Eng. C 117, 111251. 
(35) Karra, N., Nassar, T., Ripin, A. N., Schwob, O., Borlak, J., and Benita, S. (2013) Antibody conjugated PLGA nanoparticles for targeted delivery of paclitaxel palmitate: efficacy and biofate in a lung cancer mouse model. Small 9, 4221-36.

(36) Zhu, S., Li, X., Lansakara-P, D. S. P., Kumar, A., and Cui, Z. (2013) A nanoparticle depot formulation of 4-(N)-stearoyl gemcitabine shows a strong anti-tumour activity. $J$. Pharm. Pharmacol. 65, 236-242.

(37) Huang, H., Dong, Y., Zhang, Y., Ru, D., Wu, Z., Zhang, J., Shen, M., Duan, Y., and Sun, Y. (2019) GSH-sensitive Pt(IV) prodrug-loaded phase-transitional nanoparticles with a hybrid lipid-polymer shell for precise theranostics against ovarian cancer. Theranostics 9, 1047-1065.

(38) Couvreur, P., Stella, B., Reddy, L. H., Hillaireau, H., Dubernet, C., Desmaele, D., Lepetre-Mouelhi, S., Rocco, F., Dereuddre-Bosquet, N., Clayette, P., et al. (2006) Squalenoyl nanomedicines as potential therapeutics. Nano Lett. 6, 2544-2548.

(39) Desmaele, D., Gref, R., and Couvreur, P. (2012) Squalenoylation: A generic platform for nanoparticular drug delivery. J. Control. Release 161, 609-618.

(40) Couvreur, P., Reddy, L. H., Mangenot, S., Poupaert, J. H., Desmaële, D., LepêtreMouelhi, S., Pili, B., Bourgaux, C., Amenitsch, H., and Ollivon, M. (2008) Discovery of new hexagonal supramolecular nanostructures formed by squalenoylation of an anticancer nucleoside analogue. Small 4, 247-53.

(41) Reddy, L. H., Dubernet, C., Mouelhi, S. L., Marque, P. E., Desmaele, D., and Couvreur, P. (2007) A new nanomedicine of gemcitabine displays enhanced anticancer activity in sensitive and resistant leukemia types. J. Control. Release 124, $20-7$.

(42) Reddy, L. H., Khoury, H., Paci, A., Deroussent, A., Ferreira, H., Dubernet, C., Decleves, X., Besnard, M., Chacun, H., Lepetre-Mouelhi, S., et al. (2008) Squalenoylation favorably modifies the in vivo pharmacokinetics and biodistribution of gemcitabine in mice. Drug Metab. Dispos. 36, 1570-7.

(43) Reddy, L. H., Marque, P. E., Dubernet, C., Mouelhi, S. L., Desmaële, D., and Couvreur, P. (2008) Preclinical toxicology (subacute and acute) and efficacy of a new squalenoyl gemcitabine anticancer nanomedicine. J. Pharmacol. Exp. Ther. 325, 484-90.

(44) Reddy, L. H., Renoir, J. M., Marsaud, V., Lepetre-Mouelhi, S., Desmaële, D., and Couvreur, P. (2009) Anticancer efficacy of squalenoyl gemcitabine nanomedicine on 60 human tumor cell panel and on experimental tumor. Mol. Pharm. 6, 1526-35.

(45) Maksimenko, A., Mougin, J., Mura, S., Sliwinski, E., Lepeltier, E., Bourgaux, C., Lepetre, S., Zouhiri, F., Desmaele, D., and Couvreur, P. (2013) Polyisoprenoyl gemcitabine 
conjugates self assemble as nanoparticles, useful for cancer therapy. Cancer Lett. 334, 346353.

(46) Bildstein, L., Dubernet, C., and Couvreur, P. (2011) Prodrug-based intracellular delivery of anticancer agents. Adv. Drug Deliv. Rev. 63, 3-23.

(47) Dheer, D., Nicolas, J., and Shankar, R. (2019) Cathepsin-sensitive nanoscale drug delivery systems for cancer therapy and other diseases. Adv. Drug Deliv. Rev. 151-152, 130151.

(48) Kos, J., Sekirnik, A., Premzl, A., Zavasnik Bergant, V., Langerholc, T., Turk, B., Werle, B., Golouh, R., Repnik, U., Jeras, M., et al. (2005) Carboxypeptidases cathepsins X and B display distinct protein profile in human cells and tissues. Exp. Cell Res. 306, 103-13.

(49) Chen, L., Yu, Z., and Quinn, P. J. (2007) The partition of cholesterol between ordered and fluid bilayers of phosphatidylcholine: A synchrotron X-ray diffraction study. Biochim. Biophys. Acta Biomembr. 1768, 2873-2881.

(50) Pili, B., Bourgaux, C., Amenitsch, H., Keller, G., Lepêtre-Mouelhi, S., Desmaële, D., Couvreur, P., and Ollivon, M. (2010) Interaction of a new anticancer prodrug, gemcitabinesqualene, with a model membrane: Coupled DSC and XRD study. Biochim. Biophys. Acta Biomembr. 1798, 1522-1532.

(51) Vivek, K., Reddy, H., and Murthy, R. S. R. (2007) Investigations of the effect of the lipid matrix on drug entrapment, in vitro release, and physical stability of olanzapine-loaded solid lipid nanoparticles. AAPS PharmSciTech 8, 16-24.

(52) Gong, X., Moghaddam, M. J., Sagnella, S. M., Conn, C. E., Mulet, X., Danon, S. J., Waddington, L. J., and Drummond, C. J. (2011) Nanostructured self-assembly materials from neat and aqueous solutions of C18 lipid pro-drug analogues of Capecitabine - a chemotherapy agent. Focus on nanoparticulate cubosomes ${ }^{\mathrm{TM}}$ of the oleyl analogue. Soft Matter 7, 57645776.

(53) Bulanadi, J. C., Xue, A., Gong, X., Bean, P. A., Julovi, S. M., de Campo, L., Smith, R. C., and Moghaddam, M. J. (2020) Biomimetic Gemcitabine-Lipid Prodrug Nanoparticles for Pancreatic Cancer. Chempluschem 85, 1283-1291.

(54) Chapman, D., Williams, R. M., and Ladbrooke, B. D. (1967) Physical studies of phospholipids. VI. Thermotropic and lyotropic mesomorphism of some 1,2-diacylphosphatidylcholines (lecithins). Chem. Phys. Lipids 1, 445-475.

(55) Mills, T. T., Toombes, G. E. S., Tristram-Nagle, S., Smilgies, D.-M., Feigenson, G. W., and Nagle, J. F. (2008) Order Parameters and Areas in Fluid-Phase Oriented Lipid Membranes Using Wide Angle X-Ray Scattering. Biophys. J. 95, 669-681. 
(56) Chachaty, C., Rainteau, D., Tessier, C., Quinn, P. J., and Wolf, C. (2005) Building Up of the Liquid-Ordered Phase Formed by Sphingomyelin and Cholesterol. Biophys. J. 88, 4032-4044.

(57) Wu, L., Zhang, F., Chen, X., Wan, J., Wang, Y., Li, T., and Wang, H. (2020) SelfAssembled Gemcitabine Prodrug Nanoparticles Show Enhanced Efficacy against PatientDerived Pancreatic Ductal Adenocarcinoma. ACS Appl. Mater. Interfaces 12, 3327-3340.

(58) Sjöström, B., Kronberg, B., and Carlfors, J. (1993) A Method for the Preparation of Submicron Particles of Sparingly Water-Soluble Drugs by Precipitation in Oil-in-Water Emulsions. I: Influence of Emulsification and Surfactant Concentration. J. Pharm. Sci. 82, $579-583$.

(59) Venkateswarlu, V., and Manjunath, K. (2004) Preparation, characterization and in vitro release kinetics of clozapine solid lipid nanoparticles. J. Control. Release 95, 627-638.

(60) Bergman, A. M., Adema, A. D., Balzarini, J., Bruheim, S., Fichtner, I., Noordhuis, P., Fodstad, O., Myhren, F., Sandvold, M. L., Hendriks, H. R., et al. (2011) Antiproliferative activity, mechanism of action and oral antitumor activity of CP-4126, a fatty acid derivative of gemcitabine, in in vitro and in vivo tumor models. Invest. New Drugs 29, 456-466.

(61) Tao, X.-M., Wang, J.-c., Wang, J.-b., Feng, Q., Gao, S.-y., Zhang, L.-R., and Zhang, Q. (2012) Enhanced anticancer activity of gemcitabine coupling with conjugated linoleic acid against human breast cancer in vitro and in vivo. Eur. J. Pharm. Biopharm. 82, 401-409.

(62) Abu-Fayyad, A., and Nazzal, S. (2017) Gemcitabine-vitamin E conjugates: Synthesis, characterization, entrapment into nanoemulsions, and in-vitro deamination and antitumor activity. Int. J. Pharm. 528, 463-470.

(63) Jin, Y., Lian, Y., and Du, L. (2012) Self-assembly of $\mathrm{N}$-acyl derivatives of gemcitabine at the air/water interface and the formation of nanoscale structures in water. Colloids Surf. A Physicochem. Eng. Asp. 393, 60-65.

(64) Li, S., Qin, J., Tian, C., Cao, J., Fida, G., Wang, Z., Chen, H., Qian, Z., Chen, W. R., and Gu, Y. (2014) The targeting mechanism of DHA ligand and its conjugate with Gemcitabine for the enhanced tumor therapy. Oncotarget 5.

(65) Brusa, P., Immordino, M. L., Rocco, F., and Cattel, L. (2007) Antitumor Activity and Pharmacokinetics of Liposomes Containing Lipophilic Gemcitabine Prodrugs. Anticancer Res. 27, 195-199.

(66) Naguib, Y. W., Lansakara, P. D., Lashinger, L. M., Rodriguez, B. L., Valdes, S., Niu, M., Aldayel, A. M., Peng, L., Hursting, S. D., and Cui, Z. (2016) Synthesis, Characterization, 
and In Vitro and In Vivo Evaluations of 4-(N)-Docosahexaenoyl 2', 2'-Difluorodeoxycytidine with Potent and Broad-Spectrum Antitumor Activity. Neoplasia 18, 33-48.

(67) Liebmann, J. E., Cook, J. A., Lipschultz, C., Teague, D., Fisher, J., and Mitchell, J. B. (1993) Cytotoxic studies of paclitaxel (Taxol®) in human tumour cell lines. Br. J. Cancer 68, 1104-1109.

(68) Engels, F. K., Mathot, R. A., and Verweij, J. (2007) Alternative drug formulations of docetaxel: a review. Anticancer Drugs 18, 95-103.

(69) Souney, P. F., Cooper, W. D., and Cushing, D. J. (2010) PM101: intravenous amiodarone formulation changes can improve medication safety. Expert Opin. Drug Saf. 9, 319-33.

(70) Szebeni, J., Muggia, F. M., and Alving, C. R. (1998) Complement activation by Cremophor EL as a possible contributor to hypersensitivity to paclitaxel: an in vitro study. $J$. Natl. Cancer Inst. 90, 300-6.

(71) Gelderblom, H., Verweij, J., Nooter, K., and Sparreboom, A. (2001) Cremophor EL: the drawbacks and advantages of vehicle selection for drug formulation. Eur. J. Cancer 37, $1590-8$.

(72) van Zuylen, L., Verweij, J., and Sparreboom, A. (2001) Role of Formulation Vehicles in Taxane Pharmacology. Invest. New Drugs 19, 125-141.

(73) ten Tije, A. J., Verweij, J., Loos, W. J., and Sparreboom, A. (2003) Pharmacological effects of formulation vehicles : implications for cancer chemotherapy. Clin. Pharmacokinet. $42,665-85$.

(74) Schwartzberg, L. S., and Navari, R. M. (2018) Safety of Polysorbate 80 in the Oncology Setting. Adv. Ther. 35, 754-767.

(75) Griffin, W. C. (1954) Calculation of HLB Values of Non-Ionic Surfactants. J. Soc. Cosmet. Chem. 5, 249-56.

(76) Hillaireau, H., Dereuddre-Bosquet, N., Skanji, R., Bekkara-Aounallah, F., Caron, J., Lepetre, S., Argote, S., Bauduin, L., Yousfi, R., Rogez-Kreuz, C., et al. (2013) Anti-HIV efficacy and biodistribution of nucleoside reverse transcriptase inhibitors delivered as squalenoylated prodrug nanoassemblies. Biomaterials 34, 4831-4838.

(77) Lepeltier, E., Bourgaux, C., Rosilio, V., Poupaert, J. H., Meneau, F., Zouhiri, F., Lepetre-Mouelhi, S., Desmaele, D., and Couvreur, P. (2013) Self-Assembly of SqualeneBased Nucleolipids: Relating the Chemical Structure of the Bioconjugates to the Architecture of the Nanoparticles. Langmuir 29, 14795-14803. 
(78) Gaudin, A., Yemisci, M., Eroglu, H., Lepetre-Mouelhi, S., Turkoglu, O. F., DonmezDemir, B., Caban, S., Sargon, M. F., Garcia-Argote, S., Pieters, G., et al. (2014) Squalenoyl adenosine nanoparticles provide neuroprotection after stroke and spinal cord injury. Nat. Nanotechnol. 9, 1054-1062.

(79) Peramo, A., Mura, S., Yesylevskyy, S. O., Cardey, B., Sobot, D., Denis, S., Ramseyer, C., Desmaele, D., and Couvreur, P. (2018) Squalene versus cholesterol: Which is the best nanocarrier for the delivery to cells of the anticancer drug gemcitabine? C. R. Chim. 21, 974986.

(80) Vemula, P. K., Cruikshank, G. A., Karp, J. M., and John, G. (2009) Self-assembled prodrugs: an enzymatically triggered drug-delivery platform. Biomaterials 30, 383-93.

(81) Cheetham, A. G., Chakroun, R. W., Ma, W., and Cui, H. (2017) Self-assembling prodrugs. Chem. Soc. Rev. 46, 6638-6663.

(82) Wang, H., Chen, J., Xu, C., Shi, L., Tayier, M., Zhou, J., Zhang, J., Wu, J., Ye, Z., Fang, T., et al. (2017) Cancer Nanomedicines Stabilized by $\pi-\pi$ Stacking between Heterodimeric Prodrugs Enable Exceptionally High Drug Loading Capacity and Safer Delivery of Drug Combinations. Theranostics 7, 3638-3652.

(83) Allain, V., Bourgaux, C., and Couvreur, P. (2012) Self-assembled nucleolipids: from supramolecular structure to soft nucleic acid and drug delivery devices. Nucleic Acids Res. 40, 1891-1903.

(84) Sobot, D., Mura, S., Rouquette, M., Vukosavljevic, B., Cayre, F., Buchy, E., Pieters, G., Garcia-Argote, S., Windbergs, M., Desmaele, D., et al. (2017) Circulating Lipoproteins: A Trojan Horse Guiding Squalenoylated Drugs to LDL-Accumulating Cancer Cells. Mol. Ther. $25,1596-1605$.

(85) Wasan, K. M., Brocks, D. R., Lee, S. D., Sachs-Barrable, K., and Thornton, S. J. (2008) Impact of lipoproteins on the biological activity and disposition of hydrophobic drugs: implications for drug discovery. Nat. Rev. Drug Discov. 7, 84-99.

(86) Chauvin, B., Iorga, B. I., Chaminade, P., Paul, J. L., Maillard, P., Prognon, P., and Kasselouri, A. (2013) Plasma distribution of tetraphenylporphyrin derivatives relevant for Photodynamic Therapy: importance and limits of hydrophobicity. Eur. J. Pharm. Biopharm. $83,244-52$.

(87) Kratochwil, N. A., Huber, W., Müller, F., Kansy, M., and Gerber, P. R. (2002) Predicting plasma protein binding of drugs: a new approach. Biochem. Pharmacol. 64, 1355 74. 
(88) Fessi, H., Puisieux, F., Devissaguet, J. P., Ammoury, N., and Benita, S. (1989) Nanocapsule formation by interfacial polymer deposition following solvent displacement. Int. J. Pharm. 55, R1-R4.

(89) Mura, S., Buchy, E., Askin, G., Cayre, F., Mougin, J., Gouazou, S., Sobot, D., Valetti, S., Stella, B., Desmaele, D., et al. (2016) In vitro investigation of multidrug nanoparticles for combined therapy with gemcitabine and a tyrosine kinase inhibitor: Together is not better. Biochimie 130, 4-13.

(90) Vinciguerra, D., Jacobs, M., Denis, S., Mougin, J., Guillaneuf, Y., Lazzari, G., Zhu, C., Mura, S., Couvreur, P., and Nicolas, J. (2019) Heterotelechelic polymer prodrug nanoparticles: Adaptability to different drug combinations and influence of the dual functionalization on the cytotoxicity. J. Control. Release 295, 223-236. 
Table of Contents Graphic

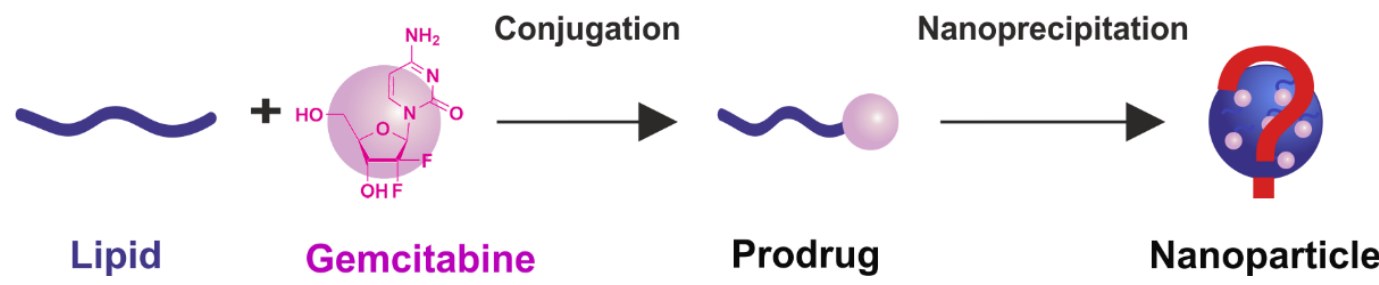

